\title{
Monopoles of the Dirac type and color confinement in QCD
}

\author{
Tsuneo Suzuki ${ }^{1, *}$, Atsuki Hiraguchi ${ }^{2, * *}$, and Katsuya Ishiguro $^{3, * * *}$ \\ ${ }^{1}$ RCNP, Osaka University, Ibaraki Osaka 567-0047, Japan \\ ${ }^{2}$ Institute of Physics, National Yang Ming Chiao Tung University, \\ Hsinchu 30010, Taiwan \\ ${ }^{3}$ Library and Information Technology, Kochi University, \\ Kochi 780-8520, Japan
}

\begin{abstract}
We present results of $S U(3)$ Monte-Carlo studies of a new color confinement scheme proposed recently due to Abelian-like monopoles of the Dirac type corresponding in the continuum limit to violation of the non-Abelian Bianchi identities (VNABI). The simulations are done without any additional gauge-fixing smoothing the vacuum. We get for the first time, in pure $S U(3)$ simulations, (1) the perfect Abelian dominance with respect to the static potentials on $(12 \sim 16)^{4}$ at $\beta=5.6-5.8$ using the multilevel method, (2) the monopole as well as Abelian dominances with respect to the static potentials by evaluating the Polyakov-loop correlators on $24^{3} \times 4$ at $\beta=5.6$. The Abelian photon part gives zero string tension. The new $S U(3)$ as well as the previous $S U(2)$ results are consistent with the new Abelian picture of color confinement that each one of eight colored electric flux is squeezed by the corresponding colored Abelian-like monopole of the Dirac type corresponding to VNABI.
\end{abstract}

\section{Introduction}

Color confinement in quantum chromodynamics (QCD) is still an important unsolved problem. As a picture of color confinement, 't Hooft [1] and Mandelstam [2] conjectured that the QCD vacuum is a kind of a magnetic superconducting state caused by condensation of magnetic monopoles and an effect dual to the Meissner effect works to confine color charges. This conjecture is very interesting, but there are many problems to be unsolved even at the present stage. Especially, to find color magnetic monopoles is not straightforward in QCD.

In gauge theories without scalar fields, it seems necessary to introduce some singularities as shown by Dirac[3] in $U(1)$ quantum electrodynamics. An interesting idea to introduce such a singularity is to project QCD to the Abelian maximal torus group by a partial (but singular) gauge fixing [4]. In $S U(3)$ QCD, the maximal torus group is Abelian $U(1)^{2}$. Then color magnetic monopoles appear as a topological object at the space-like points corresponding to the singulariry of the gauge-fixing matrix.. Condensation of the monopoles causes the dual Meissner effect with respect to $U(1)^{2}$. Numerically, an Abelian projection in various gauges such as the maximally Abelian (MA) gauge[5, 6] seems to support the conjecture[7,8].

\footnotetext{
*e-mail: tsuneo@rcnp.osaka-u.ac.jp

**e-mail: a.hiraguchi@nycu.edu.tw

***e-mail: ishiguro@kochi-u.ac.jp
} 
Although numerically interesting, the idea of Abelian projection[4] is theoretically very unsatisfactory. 1) There are infinite ways of such a partial gauge-fixing and whether the 't Hooft scheme is gauge independent or not is not known. 2) Especially, consider a Polyakov gauge in which Polyakov loops are diagonalized. In this gauge, the Abelian dual Meissner picture works good [13], where space-like monopole currents play the role of the solenoidal current squeezing the electric field. However, this fact is in contradiction with the 'tHooft idea[10].

In 2010 Bonati et al. [14] found an interesting fact that the violation of non-Abelian Bianchi identity (VNABI) exists behind the Abelian projection scenario in various gauges and hence gauge independence is naturally expected. Along this line, one of the authors (T.S.)[15] found a more general relation that VNABI is just equal to the violation of Abelianlike Bianchi identities corresponding to the existence of Abelian-like monopoles. A partial gauge-fixing is not necessary at all from the beginning. If the non-Abelian Bianchi identity is broken, Abelian-like monopoles necessarily appear due to a line-like singularity leading to a non-commutability with respect to successive partial derivatives This is hence an extension of the Dirac idea of monopoles in QED to non-Abelian QCD.

In this report, (1) the new theoretical scheme for color confinement based on the dual Meissner effect due to the above monopoles is summarized shortly. (2) The first results showing the perfect Abelian dominance and the monopole dominance in pure $S U$ (3 lattice QCD are shown next along with the short review of pure $S U(2)$ results[11]. (3) Finally numerical results showing the continuum limit of the new monopoles[16, 17] are reviewed shortly in the framework of pure $S U(2)$ lattice QCD, since existence of the continuum limit is essentially important for the new confinement scheme.

\section{Equivalence of VNABI and Abelian-like monopoles}

First of all, we prove that the Jacobi identities of covariant derivatives lead us to conclusion that violation of the non-Abelian Bianchi identities (VNABI) $J_{\mu}$ is nothing but an Abelian-like monopole $k_{\mu}$ defined by violation of the Abelian-like Bianchi identities without gauge-fixing. Define a covariant derivative operator $D_{\mu}=\partial_{\mu}-i g A_{\mu}$. The Jacobi identities are expressed as

$$
\epsilon_{\mu \nu \rho \sigma}\left[D_{v},\left[D_{\rho}, D_{\sigma}\right]\right]=0 .
$$

By direct calculations, one gets

$$
\begin{aligned}
{\left[D_{\rho}, D_{\sigma}\right] } & =\left[\partial_{\rho}-i g A_{\rho}, \partial_{\sigma}-i g A_{\sigma}\right] \\
& =-i g G_{\rho \sigma}+\left[\partial_{\rho}, \partial_{\sigma}\right],
\end{aligned}
$$

where the second commutator term of the partial derivative operators can not be discarded, since gauge fields may contain a line singularity. Actually, it is the origin of the violation of the non-Abelian Bianchi identities (VNABI) as shown in the following. The non-Abelian Bianchi identities and the Abelian-like Bianchi identities are, respectively, $D_{v} G_{\mu \nu}^{*}=0$ and $\partial_{v} f_{\mu \nu}^{*}=0$. The relation $\left[D_{v}, G_{\rho \sigma}\right]=D_{\nu} G_{\rho \sigma}$ and the Jacobi identities (1) lead us to

$$
\begin{aligned}
D_{\nu} G_{\mu \nu}^{*} & =\frac{1}{2} \epsilon_{\mu \nu \rho \sigma} D_{\nu} G_{\rho \sigma}=-\frac{i}{2 g} \epsilon_{\mu \nu \rho \sigma}\left[D_{v},\left[\partial_{\rho}, \partial_{\sigma}\right]\right] \\
& =\frac{1}{2} \epsilon_{\mu \nu \rho \sigma}\left[\partial_{\rho}, \partial_{\sigma}\right] A_{v}=\partial_{v} f_{\mu \nu}^{*}
\end{aligned}
$$

where $f_{\mu v}$ is defined as $f_{\mu v}=\partial_{\mu} A_{v}-\partial_{v} A_{\mu}=\left(\partial_{\mu} A_{v}^{a}-\partial_{v} A_{\mu}^{a}\right) \lambda^{a} / 2$. Namely Eq.(2) shows that the violation of the non-Abelian Bianchi identities, if exists, is equivalent to that of the Abelian-like Bianchi identities. 
Denote the violation of the non-Abelian Bianchi identities as $J_{\mu}=D_{\nu} G_{\mu \nu}^{*}$. An Abelianlike monopole $k_{\mu}$ without any gauge-fixing is defined as the violation of the Abelian-like Bianchi identities: $k_{\mu}=\partial_{v} f_{\mu \nu}^{*}$. Eq.(2) shows that $J_{\mu}=k_{\mu}$.

Due to the antisymmetric property of the Abelian-like field strength, we get Abelian-like conservation conditions $\partial_{\mu} k_{\mu}=0$ [18]. The Abelian-like conservation relation gives us eight conserved magnetic charges in the case of color $S U(3)$ and $N^{2}-1$ charges in the case of color $S U(N)$. But these are kinematical relations coming from the derivative with respect to the divergence of an antisymmetric tensor [18]. The number of conserved charges is different from that of the Abelian projection scenario [4], where only $N-1$ conserved charges exist in the case of color $S U(N)$.

\section{Abelian static potentials in $S U(3)$}

In $S U(2) \mathrm{QCD}$, perfect Abelian dominance is proved without performing any addtional gauge fixing using the multilevel method[12] in Ref.[9, 11]. Also perfect monopole dominance is proved very beautifully by applying the random gauge transformation as a method of the noise reduction of measuring gauge-variant quantities in the same reference[9, 11]. However, in $S U(3)$ QCD on lattice, it is not straightforward from the beginning. First, to extract Abelian link fields for all eight colors separately from non-Abelian gauge field matrix is not simple, since in $S U(3)$ the non-Abelian gauge field is not expanded by the Lie-Algebra elements in a simple way as in $S U(2)$. We choose the following method to define the Abelian link field by maximizing the following overlap quantity

$$
R A=\sum_{s, \mu} \operatorname{Tr}\left(e^{i \theta_{\mu}^{a}(s) \lambda^{a}} U_{\mu}^{\dagger}(s)\right)
$$

where $\lambda^{a}$ is the Gell-Mann matrix and no sum over $a$ is not taken. This choice in $S U(2)$ leads us to the same Abelian link fields adopted in Ref.[9, 11].

For example, we get from the maximization condition of (3) an Abelian link field $\theta_{1}(s, \mu)$ corresponding to $\lambda_{1}(S U(3)$ as

$$
\theta_{1}(s, \mu)=\tan ^{-1} \frac{\operatorname{Im}\left(U_{12}(s, \mu)+U_{21}(s, \mu)\right)}{\operatorname{Re}\left(U_{11}(s, \mu)+U_{22}(s, \mu)\right)} .
$$

Once Abelian link variables are fixed, we can extract Abelian, monopole and photon parts from the Abelian plaquette variable as follows:

$$
\theta_{\mu \nu}^{a}(s)=\bar{\theta}_{\mu \nu}^{a}(s)+2 \pi n_{\mu \nu}^{a}(s) \quad\left(\left|\bar{\theta}_{\mu \nu}^{a}\right|<\pi\right),
$$

where $n_{\mu \nu}^{a}(s)$ is an integer corresponding to the number of the Dirac string. Then an Abelian monopole current is defined by

$$
k_{\mu}^{a}(s)=-(1 / 2) \epsilon_{\mu \alpha \beta \gamma} \partial_{\alpha} \bar{\theta}_{\beta \gamma}^{a}(s+\hat{\mu}) .
$$

This definition (5) satisfies the Abelian conservation condition and takes an integer value which corresponds to the magnetic charge obeying the Dirac quantization condition[19].

\subsection{Perfect Abelian dominance in $S U(3)$}

Now let us evlaluate Abelian static potentials through Polyakov-loop correlators written by the Abelian Plaquette variable defined above:

$$
P_{\mathrm{A}}=\exp \left[i \sum_{k=0}^{N_{t}-1} \theta_{1}(s+k \hat{4}, 4)\right]
$$




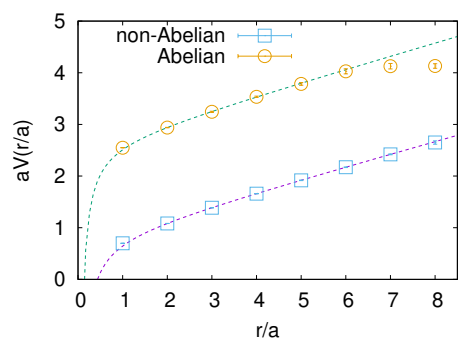

Figure 1. The static-quark potentials from non-Abelian and Abelian PLCF at $\beta=5.6$ on $16^{3} \times 16$ lattice.

Table 1. Simulation parameters for the measurement of static potential using multilevel method. $N_{\text {sub }}$ is the sublattice size divided and $N_{\text {iup }}$ is the number of internal updates in the multilevel method .

\begin{tabular}{c|c|c|c|c|c}
\hline$\beta$ & $N_{s}^{3} \times N_{t}$ & $a(\beta)[\mathrm{fm}]$ & $N_{\text {conf }}$ & $N_{\text {sub }}$ & $N_{\text {iup }}$ \\
\hline 5.60 & $16^{3} \times 16$ & 0.2235 & 6 & 2 & $10,000,000$ \\
5.70 & $12^{3} \times 12$ & 0.17016 & 6 & 2 & $5,000,000$ \\
5.80 & $12^{3} \times 12$ & 0.13642 & 6 & 3 & $5,000,000$ \\
\hline
\end{tabular}

Table 2. Best fitted values of the string tension $\sigma a^{2}$, the Coulombic coefficient $c$, and the constant $\mu a$ for the potentials $V_{\mathrm{NA}}, V_{\mathrm{A}}$.

\begin{tabular}{l|c|c|c}
\hline \multicolumn{5}{l}{$\beta=5.6,16^{3} \times 16$} \\
\hline$V_{\mathrm{NA}}$ & $0.239(2)$ & $-0.39(4)$ & $0.79(2)$ \\
$V_{\mathrm{A}}$ & $0.25(2)$ & $-0.3(1)$ & $2.6(1)$ \\
\hline \multicolumn{4}{l}{$\beta=5.7,12^{3} \times 12$} \\
\hline$V_{\mathrm{NA}}$ & $0.159(3)$ & $-0.272(8)$ & $0.79(1)$ \\
$V_{\mathrm{A}}$ & $0.145(9)$ & $-0.32(2)$ & $2.64(3)$ \\
\hline$\beta=5.8,12^{3} \times 12$ \\
\hline$V_{\mathrm{NA}}$ & $0.101(3)$ & $-0.28(1)$ & $0.82(1)$ \\
$V_{\mathrm{A}}$ & $0.102(9)$ & $-0.27(2)$ & $2.60(3)$ \\
\hline
\end{tabular}

Since the above Abelian Polyakov loop operator without any additional gauge-fixing is defined locally, the Poyakov-loop correlators can be evaluated through the multilevel method[12]. Contrary to the $S U 2$ ) case in Ref.[11], we need much more number of internal updates to get meaningful results. The simulation parameters are shown in Table1. An example of the static potentials from non-Abelian and Abelian Polyakov-loop correlators is whon in Fig. 1 The best fitted values of the non-Abelian and Abelian string tensions are plotted in Table2.

\subsection{Perfect monopole dominance in $S U(3)$}

Without adopting any further gauge fixing smoothing the vacuum, Abelian monopole static potential can reproduce fully the string tension of non-Abelian static potential in $S U(2)$ QCD as shown in Ref.[9, 11]. This is called as perfect monopole dominance of the string tension. 


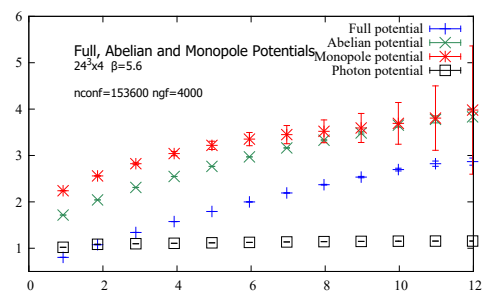

Figure 2. Full, Abelian and Monopole potentials on $24^{3} \times 4$ at $\beta=5.6$

Table 3. Simulation parameters for the measurement of the static potential and the force from $P_{\mathrm{A}}, P_{\mathrm{ph}}$ and $P_{\mathrm{mon}} . N_{\mathrm{RGT}}$ is the number of random gauge transformations.

\begin{tabular}{c|c|c|c|c}
\hline$\beta$ & $N_{s}^{3} \times N_{t}$ & $a(\beta)[\mathrm{fm}]$ & $N_{\text {conf }}$ & $N_{\text {RGT }}$ \\
\hline$S U 2,2.43$ & $24^{3} \times 8$ & $0.1029(4)$ & 7,000 & 4,000 \\
\hline$S U 3,5.6$ & $24^{3} \times 4$ & 0.2235 & 153,600 & 4,000 \\
\hline
\end{tabular}

Almost perfect monopole dominance was found in $S U(3)$ when MA gauge is adopted in Ref.[20]. However, without such additional gauge-fixing, it is found to be tremendously difficult.

We investigate the monopole contribution to the $S U(3)$ static potential through Polyakovloop correlators in order to examine the role of monopoles for confinement without any additional gauge-fixing.. The monopole part of the Polyakov loop operator is extracted as follows. Using the lattice Coulomb propagator $D\left(s-s^{\prime}\right)$, which satisfies $\partial_{v} \partial_{v}^{\prime} D\left(s-s^{\prime}\right)=-\delta_{s s^{\prime}}$ with a forward (backward) difference $\partial_{v}\left(\partial_{v}^{\prime}\right)$, the temporal component of the Abelian fields $\theta_{4}^{a}(s)$ are written as

$$
\theta_{4}^{a}(s)=-\sum_{s^{\prime}} D\left(s-s^{\prime}\right)\left[\partial_{v}^{\prime} \theta_{v 4}^{a}\left(s^{\prime}\right)+\partial_{4}\left(\partial_{v}^{\prime} \theta_{v}^{a}\left(s^{\prime}\right)\right)\right] .
$$

Inserting Eq. (7) to the Abelian Polyakov loop (6), we obtain

$$
\begin{aligned}
& P_{\mathrm{A}}^{a}=P_{\mathrm{ph}}^{a} \cdot P_{\mathrm{mon}}^{a}, \\
& P_{\mathrm{ph}}^{a}=\exp \left\{-i \sum_{k=0}^{N_{t}-1} \sum_{s^{\prime}} D\left(s+k \hat{4}-s^{\prime}\right) \partial_{v}^{\prime} \bar{\theta}_{v 4}^{a}\left(s^{\prime}\right)\right\}, \\
& P_{\text {mon }}^{a}=\exp \left\{-2 \pi i \sum_{k=0}^{N_{t}-1} \sum_{s^{\prime}} D\left(s+k \hat{4}-s^{\prime}\right) \partial_{v}^{\prime} n_{v 4}^{a}\left(s^{\prime}\right)\right\} .
\end{aligned}
$$

We call $P_{\mathrm{ph}}^{a}$ the photon and $P_{\text {mon }}^{a}$ the monopole parts of the Abelian Polyakov loop $P^{a}$, respectively [21]. The latter is due to the fact that the Dirac strings $n_{v 4}^{a}(s)$ lead to the monopole currents in Eq. (5) [19]. Note that the second term of Eq. (7) does not contribute to the Abelian Polyakov loop in Eq. (6). We show the simulation parameters and the results in comparison with one typical $S U(2)$ case.

In comparison with those in $S U(2)$ where beautiful Abelian and monopole dominances are observed using reasonable number of vacuum ensembles, we needed much more number of vacuum ensembles even on $24^{3} \times 4$ small lattice in $S U(3)$ as shown in Tabel4. Abelian dominance is seen from Abelian-Abelian Polyakov loop correlators. But in the case of 
Table 4. Best fitted values of the string tension $\sigma a^{2}$, the Coulombic coefficient $c$, and the constant $\mu a$ for the potentials $V_{\mathrm{NA}}, V_{\mathrm{A}}, V_{\mathrm{mon}}$ and $V_{\mathrm{ph}} . V_{\mathrm{FA}}\left(V_{\mathrm{FM}}\right)$ stands for the potential determined from non-Abelian and Abelian(monopole) Polyakov-loop correlators.

\begin{tabular}{c|c|c|c|c|c|c} 
& \multicolumn{7}{|c}{} \\
\hline$S U(2)$ & & $\sigma a^{2}$ & $c$ & $\mu a$ & $\mathrm{FR}(R / a)$ & $\chi^{2} / N_{\mathrm{df}}$ \\
\hline $24^{3} \times 8$ & $V_{\mathrm{NA}}$ & $0.0415(9)$ & $0.47(2)$ & $0.46(8)$ & $4.1-7.8$ & 0.99 \\
& $V_{\mathrm{A}}$ & $0.041(2)$ & $0.47(6)$ & $1.10(3)$ & $4.5-8.5$ & 1.00 \\
& $V_{\mathrm{mon}}$ & $0.043(3)$ & $0.37(4)$ & $1.39(2)$ & $2.1-7.5$ & 0.99 \\
& $V_{\mathrm{ph}}$ & $-6.0(3) \times 10^{-5}$ & $0.0059(3)$ & $0.46649(6)$ & $7.7-11.5$ & 1.02 \\
\hline \hline \multirow{2}{*}{$S U(3)$} & & & & & & \\
& & & & & & \\
& $V_{\mathrm{NA}} \times 4$ & $0.1707(47)$ & $0.855(107)$ & $1.124(45)$ & $3-10$ & 1.170 \\
& $V_{\mathrm{A}}$ & $0.1771(84)$ & $0.533(54)$ & $3.56(6)$ & $1-9$ & 0.81 \\
& $V_{\mathrm{FA}}$ & $0.1598(67)$ & $1.105(152)$ & $2.203(65)$ & $3-10$ & 1.081 \\
& $V_{\mathrm{FM}}$ & $0.146(17)$ & $0.345(29)$ & $2.477(59)$ & $0-8$ & 0.970 \\
\hline
\end{tabular}

monopole-monopole Polyakov loop correlators, we could not get good results. Since Abelian dominance is seen also from non-Abelian and Abelian Polyakov loop correlators as shown in the Tabel4., we try to study non-Abelian and monopole correlators. Since the fit is not good enough, a strong indication of monopole dominance is seen from the hybrid correlators as shown in Fig.2. When we go to larger lattice $40^{3} \times 6$ at $\beta=5.75$ (correponding to a similar temperature), Abelian dominance is seen using non-Abelian and Abelian correlators. But non-Abelian and monopole correlators are much more worse. To improve monopole static potentials for the large separation region, we may study larger $T$ lattice with much more vacuum ensembles. In the case of photon-photon correlators, the string tensions on both cases are almost zero.

\section{The continuum limit of the new Abelian-like monopoles}

Finally let us discuss an important problem if the above new-type Abelian monopoles have the continuum limit. The studies in the framework of pure $S U(2)$ QCD were done with respect to the monopole density in Ref.[16] and to the effective monopole action in Ref.[17]. Here we review the results shortly.

In both studies, it is inevitable to introduce additional gauge fixings to make the lattice vacuum smooth enough reducing the number of lattice artifact monopoles although the gauge dependence problem appear newly. It is due to the fact that even lattice artifact monopoles contribute to the monopole density or the effective monopole action equally. Hence we adopted four different smooth gauge-fixing methods to check gauge dependence, that is, MCG(maximally center gauge) [22, 23], DLCG(direct Laplacian center gauge)[24], MAWL(maximal Abelian Wilson loop gauge) [25] and MAG+U1(maximal Abelian gauge [5, 6] and $U(1)$ Landau gauge). To make the vacuum smooth, we also introduce a tadpole improved action and the block-spin transformation of monopoles[26, 27] 
Original monopoles are defined on a $a^{3}$ cube and the $n$-blocked monopoles are defined on a cube

with a lattice spacing $b=n a$ as follows:

$$
k_{\mu}^{(n)}\left(s_{n}\right)=\sum_{i, j, l=0}^{n-1} k_{\mu}\left(n s_{n}+(n-1) \hat{\mu}+i \hat{v}+j \hat{\rho}+l \hat{\sigma}\right)
$$

We considered $n=1,2,3,4,6,8,12$ blockings for $\beta=3.0 \sim 3.9$ on $48^{4}$ lattice.

We evaluated a gauge-invariant density of the $n$-blocked monopole:

$$
\rho(a(\beta), n)=\frac{\sum_{\mu, s_{n}} \sqrt{\sum_{a}\left(k_{\mu}^{(n) a}\left(s_{n}\right)\right)^{2}}}{4 \sqrt{3} V_{n} b^{3}},
$$

which is a scale-invariant quantity depending on $a(\beta)$ and $n$ generally. But if we plot $\rho$ versus $b=n a(\beta)$, we get a beautiful universal scaling function for all different gauge-fixings as shown in Fig.3. Namely we obtained clear scaling behaviors $\rho(b)$ up to the 12-step blocking transformations for $\beta=3.0 \sim 3.9$. Hence for fixed $b$, if we take $n \rightarrow \infty, a(\beta) \rightarrow 0$ that is we go to the continuum limit. The same beautiful scaling behaviors are obtained also in the case of the effective monopole action[17]. In addition to the scaling behaviors, the obtained scaling function is the same for four different gauges. Gauge independence is shown as naturally expected in the continuum limit.

Figure 3. Comparison of the Abelian-like monopole densities versus $b=n a(\beta)$ in MCG, AWL, DLCG and MAU1 cases. A uniform scaling acurve is obtained for all gauges.

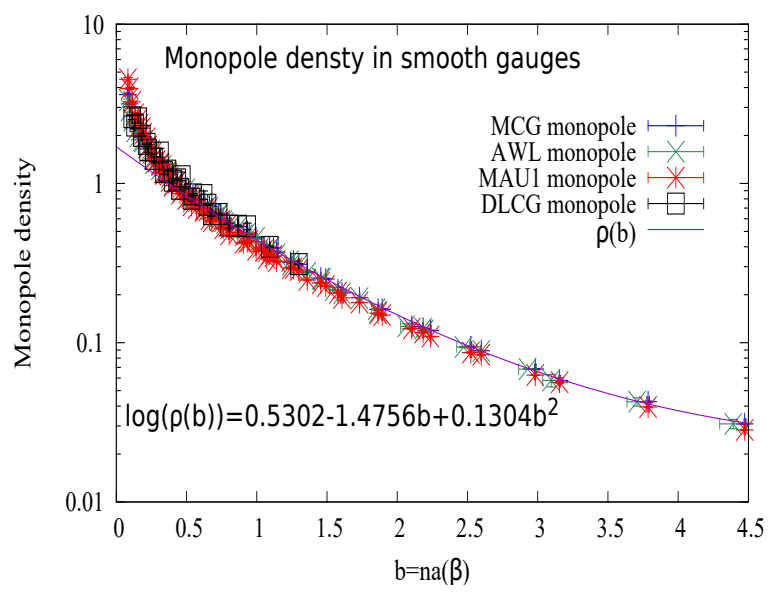

\section{ACKNOWLEDGMENTS}

The authors would like to thank Y. Koma for his computer code of the multi-level method. The numerical simulations of this work were done using High Performance Computing resources at Cybermedia Center and Research Center for Nuclear Physics of Osaka University, at Cyberscience Center of Tohoku University and at KEK. The authors would like to thank these centers for their support of computer facilities. T.S was finacially supported by JSPS KAKENHI Grant Number JP19K03848. 


\section{References}

[1] G. 't Hooft, in Proceedings of the EPS International, edited by A. Zichichi, p. 1225, 1976.

[2] S. Mandelstam, Phys. Rept. 23, 245 (1976).

[3] P. Dirac, Proc. Roy. Soc. (London) A 133, 60 (1931).

[4] G. 't Hooft, Nucl. Phys. B190, 455 (1981).

[5] A. S. Kronfeld, M. L. Laursen, G. Schierholz, and U. J. Wiese, Phys. Lett. B198, 516 (1987).

[6] A. S. Kronfeld, G. Schierholz, and U. J. Wiese, Nucl. Phys. B293, 461 (1987).

[7] T. Suzuki, Nucl. Phys. Proc. Suppl. 30, 176 (1993).

[8] M. N. Chernodub and M. I. Polikarpov, in "Confinement, Duality and Nonperturbative Aspects of QCD", edited by P. van Baal, p. 387, Cambridge, 1997, Plenum Press.

[9] T. Suzuki, K. Ishiguro, Y. Koma and T. Sekido, Phys. Rev. D77, 034502 (2008).

[10] M. N. Chernodub, Phys. Rev. D69, 094504 (2004),

[11] T. Suzuki, M. Hasegawa, K. Ishiguro, Y. Koma and T. Sekido, Phys. Rev. D80, 054504 (2009).

[12] M.Lüscher and P.Weisz, JHEP 09, 010 (2001)

[13] T. Sekido, K. Ishiguro, Y. Koma, Y. Mori, and T. Suzuki, Phys. Rev. D76, 031501 (2007).

[14] C. Bonati, A. Di Giacomo, L. Lepori and F. Pucci, Phys. Rev. D81, 085022 (2010).

[15] Tsuneo Suzuki, A new scheme for color confinement due to violation of the non-Abelian Bianchi identities, arXiv:1402.1294

[16] Tsuneo Suzuki, Katsuya Ishiguro and Vitaly Bornyakov, Phys. Rev. D97, 034501 (2018). Erratum: Phys. Rev. D97, 099905 (2018).

[17] Tsuneo Suzuki, Phys. Rev. D 97034509 (2018).

[18] J. Arafune, P.G.O. Freund and C.J. Goebel, J.Math.Phys. 16, 433 (1975).

[19] T. A. DeGrand and D. Toussaint, Phys. Rev. D22, 2478 (1980).

[20] H. Suganuma and N. Sakumichi, Proceeding of the XIIIth Quark Confinement and Hadron Spectrum, 31 July-6 August 2018, Maynooth University, Maynooth, Ireland, arXiv:1812.06827V2 (hep-lat).

[21] T. Suzuki et al., Phys. Lett. bf B347, 375 (1995).

[22] L. Del Debbio, M. Faber, J. Greensite and S. Olejnik, Phys. Rev. D55, 2298 (1997)

[23] L. Del Debbio, M. Faber, J. Giedt, J. Greensite and S. Olejnik, Phys. Rev. D58, 094501 (1998)

[24] M. Faber, J. Greensite and S. Olejnik, JHEP 111, 053 (2001).

[25] T. Suzuki et al., Nucl. Phys. Proc. Suppl. 53, 531 (1997).

[26] T.L. Ivanenko, A. V. Pochinsky and M.I. Polikarpov, Phys. Lett. B302, 458 (1993). ",

[27] H.Shiba and T.Suzuki, Phys. Lett. B351, 519 (1995). 